\begin{tabular}{|c|c|c|}
\hline 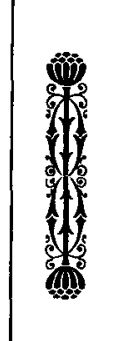 & 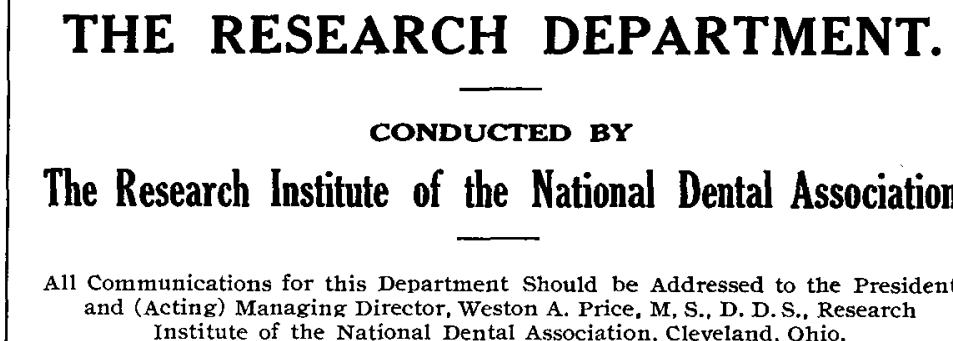 & 舜 \\
\hline
\end{tabular}

\title{
CONCERNING MOUTH INFECTIONS AS RELATED TO SYSTEMIC DISEASE.
}

\author{
THE EXPERIENCE OF THE MEDICAL CLINIC \\ (Dr. Chas. Lyman Greene, Chief of the Department of Medicine) \\ University of Minnesota.
}

By S. Marx White, B.S., M. D., Associate Professor and Assistanc Chief of Clinic in Medicine, University of Minnesota.

(This paper was prepared by Dr. White for the Research Section of Panama Prific Dental Congress at San Francisco, Sept., 1915, and is largely a discussion of the results obtained by the Research Department of the National Dental Association under the direction of Dr. Thomas B, Hartzell.)

$\mathrm{T}$

HE systematic work of the Medical Clinic in this field began two years ago with the appointment of $\mathrm{Dr}$. Trzmas B. Hartzell as Research Professor of Mouth Infections in the Medical School, and the work has becn a coöperative one. All patients, when assigned to the Medical Clinic have a preliminary examination of the mouth by the intern to determine gross evidences of alveolar or dental infection. All patients giving such evidences, together with all patients in which the presence of some focus of infection is suspected, are referred for study and relief of the Mouth conditions to the dental service.
This service consists of Dr. Hartzell and his associates, and the research work of the service is supported in part by certain specific funds set aside by the National Dental Association. Hartzell, Henrici and Leonard have already reported to this Association ${ }^{1}$ and presented other communications ${ }^{2}$ on this work. I shall refer from time to time to these communications.

Ref. 1. (Report of the Mouth Infection Research Corps of the National Dental Association.) offlcial Bulletin of the National Dental Association, Oct. 1914.

Ref. 2. (Metastatic Streptococcal Infections Arising from Primary Infections in the Neighborhood of the Human Teeth; read before the Philadelphia Academy of Stomatology, Nov. 24, 1914.) 
The aspect of this work, to which I wish to call particular attention, relates to the class of cases in our Medical wards in which evidences of mouth infection related to systemic diseases are found and to the results obtained by a systematic search for and eradication of such dental and peridental foci.

The communications of William Hunter ${ }^{3-4}$ from 1900 to 1904 appear to have been the first ones to which serious and widespread attention were paid by the medical profession, and our present awakening to the importance of this subject has served to modify only in certain details, but not in the essential principle, the ideas presented in his discussion on oral sepsis as a cause of disease in relation to general medicine. However, those who attempted to follow his argument and repeat his experience at that time met with serious difficulties and many failures, and his conclusions did not for some time. appear to be widely confirmed.

In recent years renewed interest has been aroused by the discovery of methods which reveal the presence of alveolar abscesses with an ease and directness previously impossible. Radiographic plates can be used to record the varying densities in the alveolar processes and give clear evidence as to the condition of the tooth, the presence or absence of a filling material in the dental pulp cavity, and changes occurring in the bone about the teeth. By this means alveolar abscesses have been found in the patients in whom such changes were not suspected nor could they be demonstrated readily by earlier methods. It is remarkable how free from local symptoms and signs the blind alveolar abscess may be. Röntgenographic studies of the jaws have been increasingly made in recent years and have revealed alveolar abscesses in a

Ref. 3. British Med. Jour., July, 1900, and

Ref. 4. British Med. Jour., Nor. 19, 1904. very large number of patients. Ulrich ${ }^{5}$ records the observation of 387 cases whose Röntgenographic films of the jaws were available. He states that by a conservative interpretation 736 root abscesses were seen. These were commonly multiple in any given case. Eight hundred six devitalized teeth were present, and of these 545 had blind abscesses at the tip of the roots. One hundred ninety-one abscesses were present on teeth devitalized either by accident or pulp destruction by caries. This observation as to the frequency and multiplicity of alveolar abscess is of extreme importance ant has been borne out in the main by the experience of the dental staff in our Clinic, the statistical results of which will be published by Dr. Hartzell and his co-workers.

The existence of this condition and the relative ease with which the facts can be obtained has given a tremendous impetus to the study of mouth infections within the past two or three years.

Billings $s^{6}$ and Rosenow ${ }^{7}$ have stimulated the study of such infections as related to general disease. The latter particularly by his brilliant work in growing organisms by his special methods, from joint exudates and periarticular tissues, has attracted much attention to the subject.

The study of focal infections in the mouth is only a part of the study of focal infections in general. Experience has shown that the principle sites in which chronic foci may be found are as follows:

1. The accessory nasal cavities, e. g., ethmoid, sphenoid, frontal and maxillary.

2. The middle ear and mastoid antrum.

3. The tonsils.

Ref. 5. (Streptococcicosis, read before the Minnesota Academy of Medicine, January, 1915, unpublished.)

Ref. 6. Journ. A. M. A.; LXIII, No. 23, Dec. 5, 1914.

Ref. 7. Journ. A. M. A., LXIII, Dec. 5, 1914. 
4. The alveolar processes, including all forms of dental and peridental infections.

5. The genito-urinary tract, chiefly the prostate and seminal vesicles in the male, and uterine adnexa in the female.

6. Gastro-intestinal tract, including the gall bladder, and the appendix.

Of these the accessory nasal sinuses are important, but at the present time little evidence is at hand to convict them of being the source of systemic dissemination. By this I do not mean that such dissemination has not been shown to occur, but that in our experience it is relatively infrequent.

The middle ear and mastoid antrum give prompt evidence of infection and are attacked radically and early by the surgeon.

The relation of the tonsils to the dissemination of septic infection has been increasingly recognized during the past decade and the work of Poynton and Payne and others is well known. Few clinicians at this time deny that infected tonsils are a source tho not necessarily the only source of rheumatic fe. ver, and of many of the so-called complications which are really only nonarthritic localizations, such as endocarditis, myocarditis, pericarditis, pleuritis, chorea and myositis, including polymyositis. It is, however, to the fourth group given above, i. e., the alveolar processes, including all forms of dental and peridental infections, that we are paying particular attention at the present moment.

Infection in this region may be roughly divided into two groups: (1) infections about the teeth, giving the so-called pyorrhoea pocket, and (2) abscesses and necrosis in the jaw, usually at the tips of roots, with or without sinus formation. The importance of these two groups appears to be almost equal. The recent finding of endamebae by Smith and Barrett ${ }^{8}$ in pyorrhoea confirmed by other writers gives a new aspect to this group. The discovery in emetin of a specific remedy against these parasites gives an opportunity to determine promptly whether or not the endameba is pathogenic and whether distant effects can be produced by it. The studies in our Clinics and elsewhere do not appear to me to be conclusive as yet, and much time will be required before definite conclusions can be reached. Concerning alveolar abscesses, however, considerable information is at hand.

Gilmer and Moody ${ }^{9}$ found the predominating organism to be a streptococcus, both viridans and hemolyticus being noted. Bacillus fusiformis was also reported by them, together with other organisms such as staphylococcus aureus or albus, micrococcus catarrhalis and in two instances diphtheroid bacilli.

Billings ${ }^{10}$ says: "The streptococcuspneumococcus group apparently comprise the important pathogenic bacteria related to systemic disease."

Hartzell and Henrici, working in the clinic and laboratories of the University of Minnesota, under the auspices and with funds provided by the Research Commission and science Foundation of the National Dental Association, have attempted to explain the relationship between the dental abscesses and multiple joint infections by the methods of experimental bacteriology. ${ }^{11}$

Cultures were taken from a number of

Ref. 8. Barrett, M. F., The Protozoa of the Mouth in Relation to Pyorrhoea AJveolaris. Dental Cosmos, August, 1914.

Ref. 9. A study of the Bacteriology of Alveolar Abscess and Infected Root Canals, Journ. A. M. A., LXIII, No. 23, Dec. 5, 1914.

Ref. 10. 3outh Infection as a Source of Septemic Disease, Ibid LXIII, No. 23, Dec 5, 1914.

Ref. 11. Hartzell and Henrici. A study of Streptococci from Pyorrhoea Alveolaris and from Apical abscesses, Journ. A. M. A., LXIV, No. 13, March 27, 1915. 
cases of pyorrhoea alveolaris and of dental abscess, particularly from patients suffering from acute or chronic rheumatism, Henrici isolated and classified the streptococci from pyorrhoea and alveolar abscesses according to their cultural reactions: determined their pathogenicity by animal inoculation, and in many cases prepared vaccines for treatment of patients. They state: "In the bacteriologic technic employed, our attention was directed solely to the streptococci. There were two reasons for this: first, streptococci were constantly present, and especially in apical abscesses were frequently the sole cultivable organism. Second, our immediate problem being the relationship of dental infections to rheumatism, it was thought that a study of the streptococci would be most likely to yield tangible results. Other organisms, however, were frequently noted, such as the Staphylococcus albus, the bacillus coli, Bacillus proteus, various spore-bearing aerobes of the Bacillus subtilis type, and Bacillus pyocyanceus. One recent case yielded a pure culture of the Bacillus fecalis alkaligenes. Only once was the pneumococcus obtained. Cultures have been taken from 162 cases, and in 150 of these streptococci were obtained. In seven cases where healthy teeth were extracted, using the same technic, we obtained sterile cultures."

A glance at Henrici's tables will show that the streptococcus viridans was found almost exclusively. Animal inoculations made it evident that the organisms were of low virulence but lesions developed in rabbits inoculated as follows:

Heart lesion ..........5

Kidney lesion $\ldots \ldots \ldots \ldots \ldots 7$

Aortic lesion ............ 3

Joint lesion ........... 2

of twenty-four rabbits injected. The de- tails of this cultural and animal work appear in Hartzell and Henrici's paper, but particular significance is attached to the association of heart, kidney and arterial diseases in these rabbits.

Ulrich $^{12}$ reports thirty cases of apical abscess, in all of which streptococci were found. He says: "I have purposely refrained from naming the type of streptococcus found in these abscesses. Henrici finds invariably the streptococcus viridans. In my own experience 1 have noticed early cultures show green on human blood agar, but sub-cultures on identical media very often grow haemolytic and subsequent cultures may grow less and less haemolytic. Occasionally streptococei without color or haemolysis were found."

On account of the great prevalence of streptococci in these focal lesions, Ulrich suggests the term "Streptococcicosis" to cover the class of cases in which streptococci are found in focal lesions and disseminated to other tissues.

I have reviewed the experience in our Medical Clinic from October 1, 1913, to January 1, 1915, with the purpose of showing the class of cases in which dental and peridental infections are found with evident relationship to systemic disease. I am not attempting to review here all hospital cases in which evidences of mouth sepsis were found.

The admissions to the Medical service for the period covered were 565. Of these 55 , or $9.7 \%$ of the entire admissions have been included as instances of mouth sepsis, with evident or probable relationship to systemic disease and had pyorrhoea or alveolar abscesses or both. These cases have been tabulated.

Ref. 12. Streptococcicosis, read before the Minnesota Academy of Medicine, January, 3915. 
TABLE I.

\begin{tabular}{|c|c|c|c|c|c|c|c|c|c|c|c|c|c|c|}
\hline & 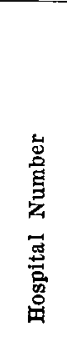 & $\stackrel{\infty}{4}$ & 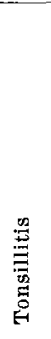 & 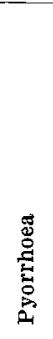 & 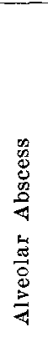 & 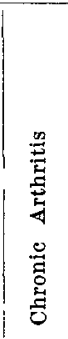 & 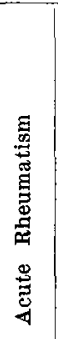 & 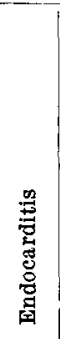 & $\begin{array}{l}\text { 总 } \\
\text { 荧 } \\
\text { 总 }\end{array}$ & 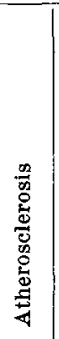 & 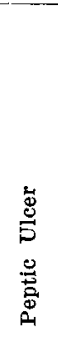 & 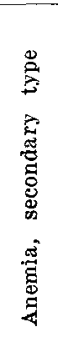 & 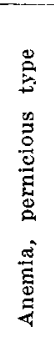 & 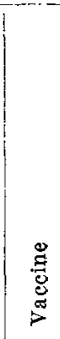 \\
\hline A. P. 1032, & 2806 & 21 & & & * & $*$ & & & & & & & & * \\
\hline Mrs. K. S. & 3077 & 53 & & & $"$ & $*$ & & $*$ & & & & & & * \\
\hline Mrs. M. B. & 3732 & 33 & & $*$ & $*$ & $*$ & & * & & & & & & * \\
\hline J. K. & 4076 & 73 & $*$ & $*$ & $*$ & 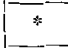 & & & $*$ & $*$ & & & & $*$ \\
\hline J. N. S. & 3937 & 39 & $*$ & * & $*$ & $*$ & & & & & & * & & $*$ \\
\hline T. G. & 3965 & 29 & & * & 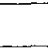 & $*$ & & $*$ & & & & * & & 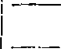 \\
\hline Mrs. L. F. & 4096 & 40 & & * & & $*$ & & $*$ & & & & & & $*$ \\
\hline N. M. & 4306 & 44 & & & * & ${ }^{*}$ & & * & & & & & & $\Rightarrow$ \\
\hline Mrs. F. W. $†$ & 3542 & 38 & & * & * & $*$ & & & & & & & & \\
\hline E. $\mathbf{R}$. & 4682 & 54 & & $*$ & & $*$ & & & * & $*$ & & & & \\
\hline Mrs. L. F. & 5496 & 28 & $*$ & & $*$ & & & & & & & & & $*$ \\
\hline Mrs. L. E. & 5847 & 38 & $*$ & & $*$ & & & & & & & & & \\
\hline Mrs. C. E. W. & 5506 & 42 & * & $*$ & $*$ & 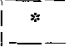 & & $*$ & & & & $*$ & & \\
\hline A. F. & 5641 & 29 & * & $*$ & $?$ & $*$ & & & & & & $*$ & & $*$ \\
\hline Mrs. I. P. & 5743 & 33 & & & $*$ & $*$ & & $*$ & & & & & & \\
\hline Mrs. A. o. & 4615 & 53 & & $*$ & * & $*$ & & $*$ & & & & & & \\
\hline
\end{tabular}

$\ddagger$ Exophthalmic goitre.

NOTES ON CASES IN TABLE I.

A. P. Hospital, No. 1032 and No. 2806. Age 21, male, white.

Clinical diagnosis:

1. Chronic tonsillitis.

2. Alveolar abscesses.

3. Chronic polyarthritis, with recurring subacute attacks.

Streptococcus hemolyticus culture from alveolar abscesses. Vaccine prepared from this. Use of vaccine followed by increase in joint soreness lasting 3 to 4 days. Improvement of general and joint symptoms. Last dose of vaccine administered January 31,1914 . Very marked improvement with great increase in joint movement and freedom from pain, except on extreme exertion. Details of this case in official Bulletin of the National Dental Association, March, 1914.

Mrs. K. S. Hospital No. 3077. Age 53, housewife. Admitted June 19, 1913. Weight 130 pounds seven years ago; average 110 ; present weight 90 .

Began to have "rheumatism" in toes and ankles 17 years ago. Affected joints have become swollen and painful. Between attacks improved but never got entirely well. During the years that followed always had pain in joints. Some 
of fingers and toes have become ankylosed.

Knees and elbows involved 15 years ago; since then elbows have become ankylosed. Severest symptoms in both knees 4 years ago. Became so painful that they could not be used and became gradually fixed. Has not been able to work for 18 months. Attempts to move joints cause intense pain and spontaneous pain, especially at night.

Four years ago began to notice weakness and shortness of breath, the latter becoming less marked after walking became impossible.

On admission she had severe pain in knees on attempting to move. Pain in hands, wrists, elbows, feet and ankles on any attempt to move. Is worse in the morning.

Examination shows marked emanciation and bony deformity. Moderate fixation of spine; pyorrhoea and dental abscesses.

Extracted all infected teeth. Made streptococcus vaccine from cultures and administered. An intercurrent attack of erysipelas occurred.

Notes show reaction about joints following injection of vaccine. In about 48 hours these reactions would subside. January 27, 1914, general condition about the same. Stiffness in elbows and wrists still exists. Finger joints more flexible than prior to vaccine. Improvement sufficient to give use of joints. Some redness and soreness of second phalangeal joint of second finger, right hand.

Patient had been encouraged to get about and use limbs, emphasis being placed on effect of vaccine in limbering up joints. Has been able to support herself by using lower limbs to get about for few feet. Ankle joints and metatarsal joints in both feet have not been ankylosed at any time and are now freely movable. Any joints which were al- most entirely ankylosed have not improved.

Discharged February 21, 1914.

Mrs. M. B. Hospital No. 3732. Age 33, female, white, American, admitted October 30, 1913.

\section{Diagnosis :} lar.)

1. Arthritis deformans (periarticu-

2. Mitral insufficiency and stenosis.

3. Anemia, (secondary type.)

4. General visceroptosis.

5. Laceration and erosion of cervix.

6. Retroversion of uterus.

7. Chronic parametritis.

8. Seborrhoea (oleosa corpora.)

9. Acne vulgaris.

10. Pyorrhoea Alveolaris.

11. Peridental abscesses.

November 9. One tooth extracted yesterday. Today complains of increased soreness and stiffness in all joints of hands and feet.

November 11. Extracted mesial root of lower left first molar-abscess; treated 7 and 8 lower right, for pyorrhoea, which was not deep at any point, but general about teeth.

November 15. Extracted mesial root and socket lower left, first molar. $\mathrm{Ab}$ scesses. Took cultureres.-Dr. Leonard.

December 18. Administered 20,000,000 streptococci vaccine. Reaction. Soreness and stiffness in joints of knee.

December 23. Recovered from vaccine and feels fair-as well as any time since admission.

December 30. Better than at any time since arrival. Wrist joints, which were immovable, are now freely movable. Tension in skin over joints of fingers, but otherwise better. The vaccines increased the soreness, stiffness and tension about the joints for two or three days; the fingers becoming quite blue and swollen, and she feels as if she hau a little fever.

January 8, 1914. "Joints much better. 
Little reaction from vaccine.-Dr. Leonard.

January 23. Patient says there has been improvement in her condition following local injection of vaccine, and she can walk with comfort instead of extreme pain as before treatment.

Laboratory report shows streptococci from dental abscesses.

More detailed report of this case in Bulletin of National Dental Association, March, 1914.

J. A. K. Hospital No. 4076. Age 73, male, white, teamster, admitted January 13, 1914.

\section{Diagnosis :}

1. Chronic tonsillitis.

2. Pyorrhoea alveolaris.

3. General adenopathy.

4. Arteriosclerosis.

5. Atheroma of aorta.

6. Hypertrophy and slight dilatation of heart.

7. Mitral regurgitation.

8. Arthritis deformans.

9. Talipes valgus.

10. Bunions.

Following are notes on dental condition: January 22, Upper teeth to be extracted; considerable pyorrhoea; no abscesses. February 3. Extracted lower incisors to get culture for vaccine, the one before having died out. February 19. Scraped lower teeth. February 26. Extracted lower right 8 th,- the only remaining dead tooth so far as could be seen. Scraped upper remaining teeth. Patient already showing improvement. March 12. Patient looks much improved. Gums almost healthy. He reports that he feels stronger.

March 23. Patient shows marked periarticular thickening across the carpal bones and including the wrist; metacarpo-phalangeal joints enlarged. Left wrist same condition, less marked than right with slightly more motion in left phalangeal joints. There is some grat- ing in both joints (wrists) and in metacarpo-phalangeal joints. Skin dry, glistening, atrophic. Percussion note over chest is normal.

March 27. Four hours after injection of vaccine felt hot and nervous. This lasted three hours and disappeared. No other symptoms noted.-H. W. W.

March 29. Moderate amount of fluid is present in both knees. H. W. W.

March 30. Effusion both knees. Right 37.5 across patella; left 36.5 . There is edema of both feet and ankles; marked deformity of toes, particularly of left foot. Large toe is markedly extended. Right foot and toes are not so deformed; no loss of motion in ankles, but some pain on pressure.

Discharged May 12, 1914, unimproved.

J. N. S. Hospital No. 3937. Age 39. Admitted December 15, 1913.

Diagnosis:

1. Chronic tonsillitis.

2. Pyorrhoea alveolaris.

3. Alveolar abscesses.

4. Chronic polyarthritis.

5. Anemia (secondary type.)

Arthritis involved knees and right elbow. Effusion into both knee joints. Marked limitation of motion in elbow. Extraction of all teeth giving radiographic evidences of abscesses. Many teeth involved.

Autogenous streptococcus viridans vaccine given. After use of vaccine would have considerable pain in region of joints and felt sick generally. Reaction completed in 48-72 hours after injection, and after fourth and fifth injections, distinct improvement noted in symptoms and pa. tient able to walk about with less pain and more comfort than he had had for two months. Small amount of exudate persists in knees.

April 7, 1914, intercurrent attack of smallpox. Returned to Hospital April 28 , with effusion somewhat increased in right knee. No effusion in left. 
April 28. Returned to Hospital for inspection.

Returned to Hospital January 9, 1915, under No. 5958. Some inflammation of tonsils. Alveolar abscess at tip of root of tooth with small area of necrosis of bone around second molar. Limitation of motion in elbow joint as on previous stay in Hospital. Effusion into both knee joints; some limitation of motion in ankles. Moderate enlargement of lymph glands in posterior cervical triangle. Abscessed teeth extracted; tonsillectomy refused.

Discharged January 21, 1915, much improved.

T. G. No. 3965. Age 29, male, white, Irish, was admitted December 19, 1913, complaining of swelling and tenderness in joints, frequent attacks of rheumatism, dyspnea, swelling of ankles.

Clinical diagnosis:

1. Chronic arthritis.

2. Secondary anemia.

3. Chronic valvular disease with mitral insufficiency.

4. Pyorrhoea alveolaris.

5. Submerged tonsils.

6. Talipes valgus.

In upper extremities marked enlargement of joints. No crepitus in joints on movement; not tender, but deformed and enlarged. Leg movement good; no ulcers. Number of small brownign red hemorrhages under skin of both legs. Skin otherwise smooth and shiny. Knee joints enlarged but not tender. Right knee smooth, larger than left; both give crepitus on movement. Somewhat warmer than surrounding parts. In right knee patellar tap present. Shows moderate distension of supra-patellar bursa, ankle joints thickened, moderately red but not tender. General adenopathy.

January 22. Seems to be no abscessed condition of teeth but considerable pyor- rhoea. Will have more radiographs taken. Made arrangements to treat case outside of Hospital (Leonard.)

Patient discharged January 22, 1914, after 35 days in the Hospital, improved.

Mrs. L. F. Hospital No. 4096. Age 40.

Marked improvement following extraction of very loose pyorrhoeal teeth and general treatment for pyorrhoea with use of vaccine. There was marked periarticular infiltration, and the improvement consisted of lessening of pain, red. ness and swelling about joints. Considerable deformity persisted. Reported dead 3 months after leaving Hospital. Cause not determined.

Mrs. F. W. Hospital No. 3542. Admitted September 24, 1913. Age 38 .

Diagnosis :

1. Exophthalmic goitre.

2. Chronic arthritis.

3. Pyorrhoea alveolaris.

4. Alveolar abscesses.

Extraction of multiple abscess while in Hospital. Pyorrhoea treated. Marked improvement in joint condition and in symptoms of hyperthyroidism. Pulse at time of discharge varied from 80 to 112 per minute; average 90 . Discharged November 4, 1914, much improved.

N. M. Hospital No. 4306. Age 44.

Clinical diagnosis:

1. Multiple alveolar abscesses.

2. Chronic arthritis.

3. Chronic valvular disease with mitral insufficiency.

Impossible to determine whether the marked improvement which occurred was due to removal of foci or use of vaccine. Results of vaccine therapy were not apparent. There was marked periarticular thickening, and altho joint symptoms improved, considerable deformity remained. 
Mrs. E. R. Hospital No. 4682. Age 54. Clinical diagnosis:

1. Pyorrhoea alveolaris.

2. Chronic multiple.

3. Chronic diffuse nephritis.

4. Hypertension and atherosclerosis.

Very marked pyorrhoea with great loosening of teeth. May $16 \mathrm{th}$ was made very much more lame and ill as result of stirring up infection in the mouth on the 14th. June 4, 2 remaining teeth extracted. Not much systemic reaction. Very great improvement following cleaning out of mouth sepsis. Considerable deformity remains.

Mrs. L. F. Hospital No. 5496. Age 28. Admitted October 7, 1914.

Clinical diagnosis:

1. Chronic tonsillitis (tonsillectomy October 17, 1914.)

2. Alveolar ábscesses.

3. Chronic rheumatoid arthritis.

4. Hypoplasia of uterus.

Multiple alveolar abscesses found. Extraction. Streptococci cultivated and autogenous streptococcus vaccine given. Marked reaction about joints after use of vaccine. February 10, became melancholly; later stupid. Temperature varied from 99-101. Became gradually weaker and died March 20, of broncho-pneumonia. Autopsy by Dr. W. C. Johnson.

Autopsy diagnosis adds:

5. Acute vegatative endocarditis.

6. Acute interstitial myocarditis.

7. Broncho-pneumonia.

8. Infantile uterus and ovaries.

Mrs. L. E. Hospital No. 5847. Age 32, Polyarthritis without deformity, involvinf sacroiliac synchondrosis, left knee joint and tibiofibular articulation. Sciatica. The sciatic pains disappeared immediately and entirely when abscessed teeth were removed, and pain over sacroiliac synchondrosis disappeared. Other joint symptoms subsided.
Mrs. C. E. W. Hospital No. 5506. Age 42.

Clinical diagnosis:

1. Chronic tonsillitis.

2. Pyorrhoea alveolaris.

3. Alveolar abscesses.

4. Chronic polyarthritis.

5. Chronic valvular disease.

6. Secondary anemia.

Uterine fibroids. Subtotal hysterectomy performed with good recovery. Patient would permit removal of two $a b$ scessed teeth remaining after a number of other abscessed teeth were extracted. No tonsillectomy performed. Patient left Hospital against advice of staff, condition distinctly improved.

A. F. Hospital No, 5641. Age 29.

Clinical diagnosis:

1. Chronic tonsillitis.

2. Pyorrhoea alveolaris.

3. Anemia (secondary type.)

4. Polyarthritis (rheumatoid type) with moderate periarticular infiltration. First attack shows distinct infiltration of periarticular tissues with reddening and great tenderness.

Areas of rarefaction about one tooth root, but no evidence of infection on extraction. Pyorrhoea considered by dental service to be possible focus of infection. After treatment for pyorrhoea had an acute exacerbation of joint trouble beginning 24 hours and lasting 48 hours. Tonsillectomy was performed, after which gradual but persistent and permanent improvement occurred.

Mrs. I. P. Hospital No. 5743. Age 33. Clinical diagnosis:

1. Alveolar abscesses.

2. Chronic arthritis of hip.

3. Acute dry pleurisy.

4. Chronic bronchitis.

5. Fetal adenoma of thyroid.

Would not allow eradication of mouth foci. 
Mrs. A. O. Hospital No. 4615. Age 53. ticular thickening in many joints, MarkClinical diagnosis:

1. Pyorrhoea alveolaris.

2. Alveolar abscesses.

3. Chronic valvular disease.

4. Chronic polyarthritis.

Entered Hospital with marked periared limitation of motion. Pain on pres sure. Mitral and aortic insufficiency. Pyorrhoea and miltiple abscesses. Removal of mouth foci was followed by marked lessening of tenderness and pain on motion. There have been no acute attacks since.

TABLE II.

\begin{tabular}{|c|c|c|c|c|c|c|c|c|c|c|c|c|c|c|}
\hline & 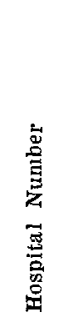 & $\underset{d}{0}$ & 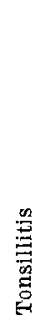 & 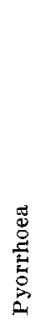 & 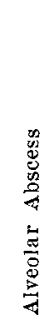 & 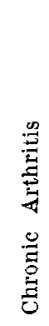 & 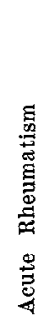 & 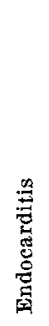 & 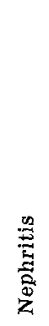 & 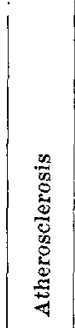 & 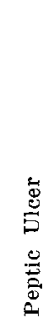 & 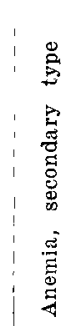 & 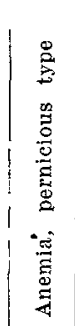 & 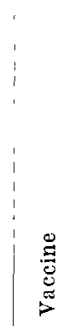 \\
\hline A. A. & 4936 & 31 & $*$ & & $*$ & $*$ & & $*$ & & & & & & \\
\hline E. $S$. & 4393 & 25 & & & 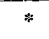 & & $*$ & $*$ & & & & & $\%$ & \\
\hline J. B. S. & 4686 & 40 & & $*$ & $*$ & & $*$ & * & $*$ & & & & & \\
\hline E. $\mathrm{G}$. & $402 T$ & 20 & & $*$ & & & * & $*$ & & & $*$ & $*$ & & \\
\hline J. B. & 5909 & 37 & $*$ & $*$ & $*$ & & $*$ & * & & & & & & \\
\hline D. E. S. & 6028 & 29 & $*$ & & $*$ & & $*$ & $*$ & & & & & $\mid$ & \\
\hline A. $\mathrm{K}$. & 4140 & 38 & & $*$ & * & & $*$ & & & & & & $*$ & \\
\hline Mrs. M. B. & 5514 & 26 & & $*$ & $*$ & & & $*$ & & & & & & \\
\hline Mrs. B. L $\dagger$ & 4271 & 31 & * & & $*$ & & & $*$ & & & & & & \\
\hline Mrs. M. S. & 4324 & 52 & $*$ & $*$ & & & & $*$ & & & * & & & \\
\hline E. $\mathbf{R}$. & 3605 & 33 & & $*$ & & & & * & * & & & & & \\
\hline Mrs. J. B. & 4954 & 75 & & $*$ & $*$ & & & & * & i & & & 1 & \\
\hline J. $\mathbf{O}^{\prime} \mathbf{B}$ & 4968 & 63 & & $*$ & & & & & * & I * & $*$ & & & \\
\hline F. $\mathrm{M}$. & $54 \overline{7}$ & 36 & $*$ & $*$ & & & & & * & $1 *$ & $*$ & & & \\
\hline J. $\mathbf{s}$. & 5365 & 67 & & * & & & & & $*$ & 1 & & & $*$ & \\
\hline F. J. & 5635 & 48 & & & $*$ & & & $*$ & $*$ & $1 *$ & $*$ & & 1 & \\
\hline F. L. H. & 5556 & 63 & & $*$ & $*$ & & & $*$ & & $*$ & * & & & \\
\hline M. C. & 4247 & 22 & & & $*$ & & & & * & $*$ & * & & I & \\
\hline N. W. & 5576 & 56 & & & $*$ & & & & $*$ & 1 & $*$ & & & \\
\hline Mrs. R. B. & 4438 & 39 & & $*$ & & & & & * & & $*$ & & & \\
\hline N. S. & 4892 & 50 & & $*$ & $*$ & & & & $*$ & $1 *$ & $*$ & & & \\
\hline
\end{tabular}

†Iritis, facial neuralgia. 


\section{NOTES ON CASES IN TABLE II.}

A. A. Hospital No. 4936. Male, age 31. Clinical diagnosis:

1. Chronic tonsillitis.

2. Alveolar abscesses.

3. Acute rheumatism.

4. Endocarditis.

5. Syphilis (positive Wassermann.)

Marked soreness and stiffness of joints quite persistent. No periarticular thickening. Marked improvement of symptoms following eradication of areas of peridental infection. Cultures from dental abscesses gave streptococcus viridans and bacillus coli.

E. S. Hospital No. 4393. Male, age 25. Clinical diagnosis:

1. Alveolar abscesses.

2. Acute rheumatism.

3. Chronic valvular disease.

Pericarditis with effusion; ulcer of esophagus and cardiospasm. Marked improvement followed extraction of abscessed teeth. There was temperature reaction after first injection of vaccine. Left Hospital symptomatically well.

J. B. B. Hospital No. 4686. Male, age 40 .

Clinical diagnosis:

1. Pyorrhoea alveolaris.

2. Alveolar abscesses.

3. Acute rheumatism.

4. Acute endocarditis and myocarditis.

5. Acute nephritis.

Multiple dental abscesses had existed for years. No evidence of tonsil infection. Heart sounds showed triple rhythm, and electrocardiographic tracings show increase of As-Vs interval to $3-10 \mathrm{sec}$ onds. Died. No autopsy allowed. 20.

E. G. Hospital No. 4027. Male, age

Clinical diagnosis:

1. Pyorrhoea alveolaris.
2. Acute rheumatism.

3. Chronic and acute endocarditis.

4. Peptic ulcer.

5. Anemia (secondary type.)

6. Former gastroenterostomy with patulous opening.

7. Diabetes mellitus.

Treatment of pyorrhoea began promptly. Patient had a prompt subsidence of the rheumatic symptoms after admission, and February $1,1914,30$ days after admission to the Hospital, he had hematemesis with considerable loss of blood. Five days later stools still showed a small amount of occult blood. The sugar, which had disappeared before hematemesis, appeared in small quantity imme. diately following it. Absent since. Marked improvement of all symptoms.

J. B. Hospital No. 5909. Male, age 37. Clinical diagnosis:

1. Chronic tonsillitis.

2. Pyorrhcea alveolaris.

3. Alveolar abscesses.

4. Acute rheumatism.

5. Chronic valvular disease with mitral insufficiency.

6. Syphilis (positive Wassermann.)

Marked improvement promptly followed removal of alveolar abscesses. Discharged after one month, much improved. No anti-syphilitic treatment given.

D. E. S. Hospital No. 6028. Male, age 29 .

Had injury, breaking 3 ribs, 10 weeks previous to admission. Was in another Hospital for eight days, then home. A month after injury attempted to go to work, but was found to be coming down with acute pneumonia. During convalescence from pneumonia, stiffness and pain in joints appeared with swelling after a few days. An attack of acute rheumatism developed, and he was brought to the University Hospital in this condition. No history of infection 
of tonsils preceding rheumatism, but evidences of active tonsillitis were found on admission. Eight abscesses were found at roots of teeth.

Extraction of abscessed teeth proceeded with cautiously. Tonsillectomy was finally advised and performed five weeks after admission. Considerable hemorrhage followed this. Subsequent improvement rapid and continuous.

A. K. Hospital No. 4140. Age 38, male, white, American, laborer, was admitted to the University Hospital January 26, 1914, complaining of swelling of right wrist, left ankle, aching and soreness in infected joints, sharp pain in right chest passing away after few short breaths, weakness, and sleeplessness.

Examination of extremities shows tenderness in right shoulder and elbow; right wrist and hand very tender, and wrist distinctly swollen. Moderate swelling below external malleolus. Left ankle and left foot distinctly tender.

Examination of mouth shows teeth of upper right-2nd, $3 \mathrm{~d}$ and 8 th--dead, and probably septic; upper left first, also some pyorrhoea and much filthy accumulation on gum margin. Disagreeable odor from mouth. (Leonard.)

February 3. Extracted upper left central incisors and right lateral incisors for cultures for vaccine. Streptococcus viridans.

February 14. Could not extract right lateral $3 \mathrm{~d}$ so cut it out with burr, euretting a large abscessed sinus at the same time.

\section{Diagnosis :}

1. Pyorrhoea alveolaris.

2. Dental abscesses.

3. Pyorrhoea alveolaris.

4. Glandular adenopathy.

5. Acute rheumatism.

Mild reactions followed use of vaccine.

He was discharged February 26, 1914, much improved.
Mrs. M. B. Hospital No. 5514. Age 26. American, white, admitted October 8, 1914.

Clinical diagnosis :

1. Pyorrhoea alveolaris.

2. Alveolar abscesses.

3. Chronic valvular disease with mitral stenosis.

4. Achylia gastrica.

5. Asthenia.

Marked improvement, particularly of neurasthenic symptoms, following dental work. Patient died not allowing comple. tion of all work, altho the abscesses were removed. Pyorrhoea was not completely eradicated.

Mrs. B. L. Hospital No. 4271. Age 31.

Diagnosis:

1. Chronic tonsillitis.

2. Alveolar abscesses.

3. Chronic valvular disease of heart.

4. Iritis.

5. Facial neuralgia.

Remarkable improvement with complete disappearance of iritis and neuralgia after removal of teeth and tonsillectomy.

Mrs. M. S. Hospital No. 4324. Age 52. Clinical diagnosis:

1. Chronic tonsillitis.

2. Pyorrhoea alveolaris.

3. Chronic valvular disease of heart.

4. Anemia (secondary type.)

Much joint soreness and pain -without swelling or redness. Joint pains decidedly improved. April 1, 1915, states that she has had no acute attack of joint pains since leaving the Hospital.

E. R. Hospital No. 3605. Age 33, male. Admitted October 4, 1913.

Clinical diagnosis:

1. Pyorrhoea alveolaris and ulcerative stomatitis (fusiform bacillus and spirillum of Vincent.) Streptococc found in pyorrhoea pockets. 
2. Chronic valvular diseases with aortic insufficiency, mitral insufficiency and stenosis.

3. Passive congestion of lungs, liver, and spleen.

4. Ascites.

5. Chronic interstitial nephritis.

6. Purpura (over both legs.)

7. Secondary anemia.

Note by Dr. Hartzell. "In mouth, bone is necrotic in the lower arch on all sides from central back for a depth of $1 / 8$ inch.

Died October 21, 1913.

Autopsy diagnosis confirms above, adding (8) lobar pneumonia; (9) Chronic pleuritis; (10) Infarcts of spleen and kidneys.) Streptococci found in heart blood. Microscopically, foci of infiltration in myocardium.

Mrs. J. C. Hospital No. 4954. Age 75.

Gastroptosis, nephritis and severe mouth sepsis with pyorrhoea, multiple alveolar abscesses and ulcerative stomatitis. Marked reduction of kidney excretion, as shown by phenol sulphon phthalein test. Remained only 21 days and little improvement seen. 63.

J. O. B. Hospital No. 4968. Male, age

Clinical diagnosis:

1. Pyorrhoea alveolaris.

2. Chronic nephritis.

3. Arteriosclerosis.

4. Secondary anemia,

5. Achylia gastrica.

6. Chronic colitis.

The pyorrhoea was extreme and there were many dead pulps. Dental service advised complete extraction. This was done September 22, 1914. Discharged October 23, 1914, greatly improved. Improvement has persisted, altho he relapsed twice after residence in Hospital before this.
F. M. Hospital No. 5447. Male, age 36, white. Was admitted February 28, 1914.

Clinical diagnosis :

1. Chronic tonsillitis.

2. Pyorrhoea alveolaris.

3. Chronic nephritis.

4. Arterial hypertension and sclerosis.

5. Cardiac hypertrophy.

6. Passive congestion of viscera.

7. Anemia (secondary type.)

General care given and pyorrhoea radically treated by dental service. Prompt improvement occurred and he was discharged December 16, 1914, greatly improved.

J. S. Hospital No. 5365. Male, age 67, admitted February 14, 1914.

Clinical diagnosis:

1. Pyorrhoea alveolaris.

2. Chronic nephritis.

3. Anemia (pernicious type.)

4. Hypertrophy and dilatation of heart with mitral insufficiency.

Pyorrhoea treated radically by dental service, apparently without effect. Died November 3, 1914. Autopsy not permitted.

F. J. Hospital No. 5635. Male, age 55.

Clinical diagnosis:

1. Alveolar abscesses.

2. Chronic valvular disease with mitral and aortic insufficiency.

3. Chronic nephritis.

4. Atherosclerosis.

5. Secondary anemia.

6. Chronic bronchitis.

Foci of mouth sepsis were cautiously attacked by dental service and finally eradicated. Moderate improvement.

F. L. H. Hospital No. 5556. Age 63.

Entered Hospital with chronic valvular disease, decompensation of heart, 


\section{aroperit: \\ Dento \\ tor Contrit. \\ MOUTH INFECTIONS AS REIJATED TO SWSTEMIE DISEASE. $\because 275$}

ascites and anasarca. Multiple alreolar abscesses found and treated by extraction. Death 77 days after admission. Autopsy by Dr. W. C. Joknson.

Autopsy diagnosis:

1. Cardiac hypertrophy and dilatation.

2. Chronic endocarditis-aortic and mitral.

3. Atheroma and dilatation of aorta.

4. Thrombosis of right auricle.

5. Atheroma of coronary arteries.

6. Chronic pericarditis.

7. Pulmonary infarct.

8. Hemothorax.

9. Chronic pleuritis.

10. Healed miliary tuberculosis of spleen.

11. Passive congestion of lungs, spleen and kidneys.

12. Meckel's diverticulum.

M. C. Hospital No. 4247. Age 22, female.

Clinical diagnosis:

1. Chronic nephritis.

2. Splenic anemia (?) with splenomagaly.

3. Hypertrophy of heart.

4. Atherosclerosis.

5. Alveolar abscesses.

Would not allow extraction. February 20,1914 , hemoglobin $46 \%$; r. b. c. 3,000 ,000; leucocytes 3600. April 23, 1914, hemogloblin $35 \%$; r. b. c. $2,768,000$; leucocytes 2840 . December 17, 1914, hemoglobin $38 \%$; r. b. c. $2,500,000$; leucocytes 2500 .

Condition unimproved.

N. W. Hospital No. 5576. Age 56 , male.

Marked pyorrhoea; numerous dental abscesses. Has a marked nephritis with moderate reduction of excretion of phenol sulphon phthalein in two hours. Improvement moderate after elimination of mouth sepsis. Albumen persists in traces. Moderate secondary anemia.
Mrs. R. B. Hospital No. 4438. Age 39, Jewish, white, admitted April 2, 1914.

\section{Diagnosis :}

1. Secondary anemia (high grade.)

2. General pigmentation.

3. Slight cardiac dilatation.

4. Visceroptosis.

5. Albuminuria.

6. Chronic (nasal septum) ulcer.

7. Diastasis recti.

8. Asthenia.

9. Pyorrhoea alveolaris.

10. Enlargement of thyroid (moderate grade.)

Dental radiograph shows upper left central and lateral roots unfilled, with slight rarified area at end of lateral. Also shows cuspids impacted with crowns pointing mesially and lying horizontally. (Dr. Leonard.)

Pyorrhoea treated radically. Patient responded very well to rest in bed and iron, her hemoglobin rising from $25 \%$ to $67 \%$. Sixty-five per cent was estimated the 27th of May. Patient left Hospital June 3, 1914, much improved.

Note by Dr. Ulrich. "One of the interesting features of her case was that there were points suggesting congenital hemolytic jaundice; but testing the resistance of red blood corpuscles dismissed this supposition, and it was shown to be an ordinary case of secondary anemia."

N. S. Hospital No. 4882. Male, age 50, admitted June 19, 1914.

Clinical diagnosis:

1. Pyorrhoea alveolaris.

2. Alveolar abscesses.

3. Chronic nephritis.

4. Atherosclerosis.

5. Hypertrophy of heart.

6. Chronic passive congestion of viscera.

7. Anemia (secondary type.)

Died November 28, 1914. Autopsy diagnosis by Dr. W. C. Johnson. 
Autopsy diagnosis:

1. Chronic interstitial nephritis with multiple renal adenomata.

2. Cardiac hypertrophy and dilatation.

3. Chronic endocarditis.

4. Acute fibrinous pericarditis.

5. Chronic pericarditis.
6. Atheroma of aorta.

7. Edema of lungs.

8. Congestion of spleen and chronic perisplenitis.

9. Slight passive congestion of liver.

10. Chronic interstitial pancreatitis.

11. Ascites.

12. Edema.

TABLE III.

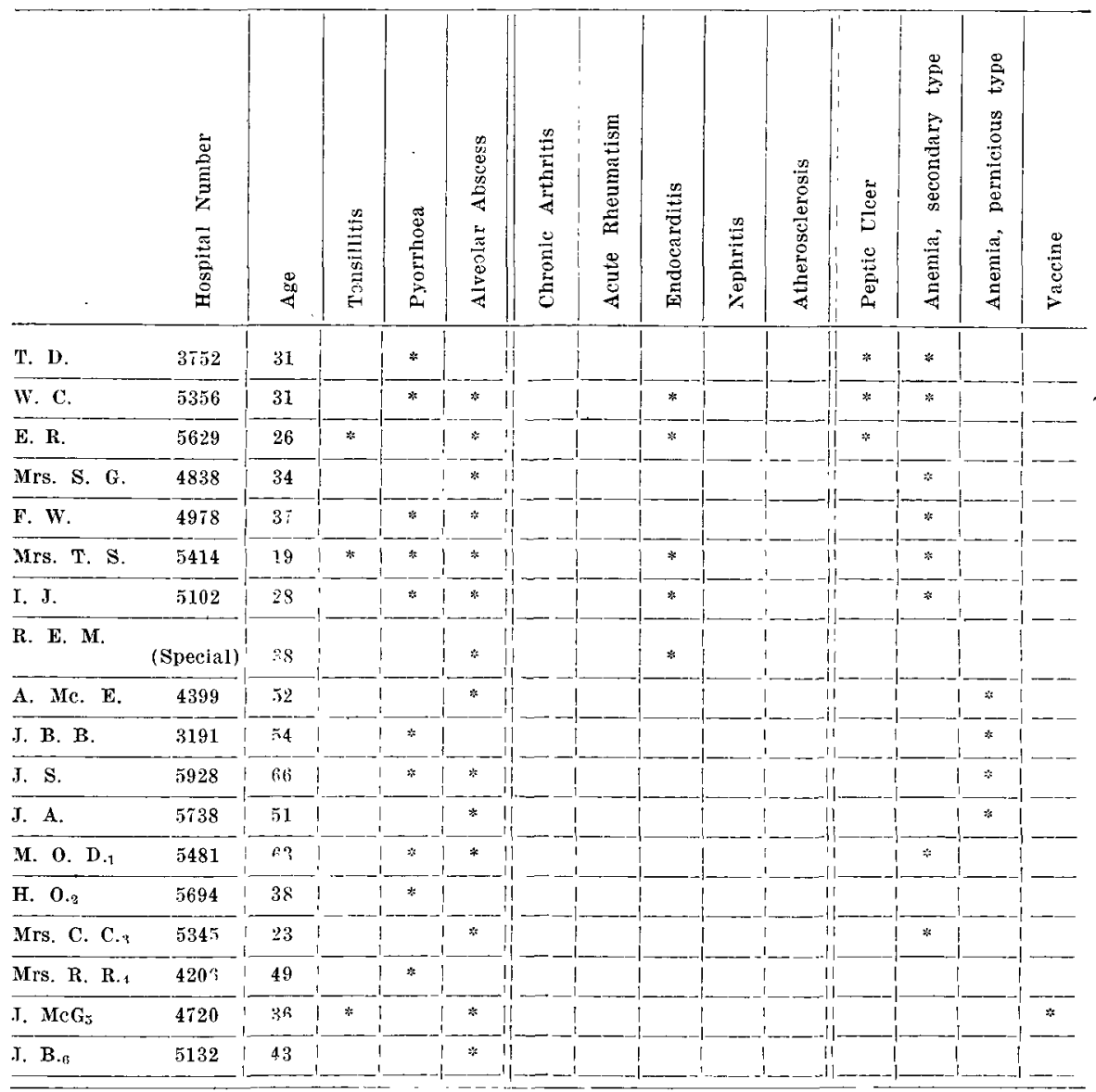

Chronic eholecystitis.

Hypochlorhydria with persistent diarrhoea.

"Multiple neuritis, pelvic cellulitis (old.)

Asciatic neuritis (bilateral.)

-Sinusitis (ethmoidal Bronchial asthma.)

.Lobar pneumonia delayed resolution. 
NOTES ON CASES IN TABLE III.

T. D. Hospital No. 3752. Age 45, male, white, admitted November 3, 1913.

Clinical diagnosis:

1. Pyorrhoea alveolaris.

2. Peptic ulcer.

3. Anemia (secondary type.)

Treated by rest in bed, feeding every two hours, followed by administration of alkalis and ferrous carbonate.

Notes by dental service show marked mouth sepsis, and pyorrhoea was treated radically. Discharged December 23 , with mouth in very good condition. Gastric symptoms have disappeared and patient has remained well.

W. C. Hospital No. 5356. Age 31, male.

Diagnosis :

1. Pyorrhoea alveolaris.

2. Alveolar abscesses.

3. Chronic valvular disease with mitral insufficiency.

4. Peptic ulcer.

5. Anemia (secondary type.)

Mouth sepsis was thoroly eradicated. Rapid improvement. Reappeared for examination four months after dismissal. States that before admission he was troubled almost continuously with stomach symptoms, but since leaving the Hospital has been absolutely free and never had so long a period of freedom from symptoms before.

E. R. Hospital No. 5629. Male, age 26.

Clinical diagnosis:

1. Gastric ulcer.

2. Mitral insufficiency.

3. Chronic tonsillitis (tonsillectomy.)

4. Alveolar abscesses (extraction while in Hospital.)

Treatment in this case is that usually given peptic ulcer cases. Improvement after eliminating foci of infection was very rapid. He has remained well.
Mrs. S. G. Hospital No. 4828. Jewish, age 34 , female, white, admitted June 9, 1914.

Clinical diagnosis :

1. Dental caries and alveolar abscesses.

2. Secondary anemia.

3. Asthenia.

Mouth sepsis eradicated. Moderate improvement in general condition. Hemoglobin 6-20-14, 67\%; 7-17-14, 85\%. Iron carbonate administered while in the Hospital.

F. W. Hospital No. 4978. Female, age 37 , admitted July 7, 1914 .

Clinical diagnosis:

1. Pyorrhoea alveolaris.

2. Alveolar abscesses.

3. Albuminuria.

4. Anemia (secondary type.)

Mouth sepsis eradicated by extraction of all remaining teeth and many septic roots. Marked improvement.

Mrs. T. S. Hospital No. 5415. Female, age 19.

Clinical diagnosis:

1. Chronic tonsillitis.

2. Pyorrhoea alveolaris.

3. Alveolar abscesses.

4. Chronic valvular disease.

5. Anemia (secondary type.)

Came to Hospital because of anemia. Anemia began to improve before elimination of alveolar abscesses attempted. Dental treatment did not exert demonstrable influence in general improvement, which was rapid.

I. J. Hospital No. 5102. Female, age 28.

Clinical diagnosis:

1. Pyorrhoea alveolaris.

2. Dental abscesses.

3. Chronic valvular disease.

4. Anemia (secondard type.)

5. Syphilis (?)

Wassermann reaction negative, but 


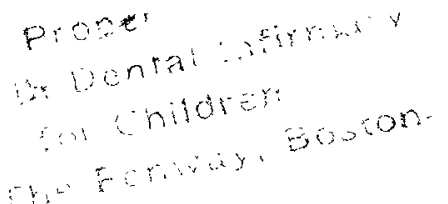

patient had been taking mercurial treatment before admission.

Notes by dental service record extensive infection of teeth. Undetermined whether possible syphilitic infection or mouth sepsis responsible for condition.

R. E. M. Special. Male, age 36, white. Clinical diagnosis:

1. Alveolar abscesses.

2. Chronic valvular disease with aortic and mitral insufficiency.

3. Auricular fibrillation.

Extraction of all these infected teeth done at one time, against advice of staff, outside of Hospital. Patient returned with marked sepsis of jaws and in a very serious condition. Was in bed two weeks, but a month was required before he was able to return to work.

A. McE. Hospital No. 4399. Male, age 52. Admitted March 21, 1914.

Clinical diagnosis:

1. Pernicious anemia.

2. Alveolar abscesses, upper right central, lower right third molar, lower left third molar.

Alveolar abscesses treated by extraction of teeth involved.

\section{BLOOD COUNTS.}

March 22, 1914, r. b. c. 1,100,000; hemogloblin 18\%; leucocytes 4500; marked poikilocytosis and anisocytosis. Normoblasts and megaloblasts present. Lymphocytes 38.5; large mononuclear $1 \%$; transitional 0\%; Polymorphonuclear, $60 \%$; eosinophile $0 \%$; basophile $5 \%$.

April 2, 1914, r. b. c. 900,000; hemoglobin $15 \%$.

April 8, 1914, r. b. c. 700,000 ; hemoglobin $21 \%$.

April 18, 1914, r.b.c. 1,300,000; hemoglobin $31 \%$.

He left the Hospital against advice of the staff. Patient reported for examination September 9, 1914. He stated that he felt well except shortness of breath and swelling of legs. On examination, a fair amount of edema of legs between the knee and ankle found, but practically absent below. Showed a moderate degree of emanciation; skin yellowish brown color; liver margin at umbilical level. Heart moderately dilated. Marked systolic thrill over entire heart and up into carotids. Well marked diastolic murmur transmitted down left side of sternum.

J. B. B. Hospital No. 3191. Male, age 54 .

First admission July 3,1913 , to July 18, 1913. Fifteen days in Hospital. On admission, hemoglobin $40 \%$; r. b. c. 1,240,000. On discharge, hemoglobin $38 \%$; r. b. c. $1,750,000$. Left Hospital against advice of attending physician. Readmitted May 27, 1914 (second admission No. 3346) to August 8, 1914. October 20 , blood on admission: hemoglobin $72 \%$; r. b. c. $3,736,000$. Trichomonas intestinalis found in stool August 24. Disappeared promptly from stool following use of liquor potassi arsenitis and dilute hydrochbric acid and pepsin. On both of these visits to Hospital, pyorrhoea was found and oral hygiene used. Readmitted July 28, 1914. Blood examination: Hemoglobin $30 \%$; r. b. c. 1,600,000. On discharge, hemoglobin $69 \%$; r. b. c. $3,900,000$. Dental service found no evidence of abscesses. Moderate gingivitis. Mouth responded promptly to dental treatment.

J. S. Hospital No. 5074 and No. 5928. Age 66, male.

Clinical diagnosis :

1. Pyorrhoea alveolaris.

2. Alveolar abscesses.

3. Anemia (pernicious type.)

Blood examination August 4, 1914: Hemoglobin $30 \%$; r. b. c. $1,200,000$. November 5, 1914, hemoglobin $37 \%$; r. b. c. 
1,900,000. Moderate improvement after clearing out of mouth sepsis. Patient returned after 6 weeks. January 6,1915 , hemoglobin $32 \%$; r. b. c. 1,200,000. January 18 , 1915, hemoglobin $37 \%$; r. b. c. 2,000,000. Left Hospital condition much improved. During second residence in Hospital, pyorrhoea but no abscesses found.

\section{J. A. Hospital No. 5738. Male, age 51.}

A typical case of pernicious anemia. Four teeth with large alveolar abscesses and much pyorrhoea. Radical treatment. December 2, 1914, hemoglobin 18\%; r. b. c. 1,100,000; leucocytes 300. February 4, 1915, hemoglobin $54 \%$; r. b. c. 2,400 , 000 .

M. O. D. Hospital No. 5481. Male, age 63, admitted October 4, 1914.

Clinical diagnosis:

1. Pyorrhoea alveolaris.

2. Alveolar abscesses.

3. Chronic cholecystitis.

4. Anemia (seconđary type.)

Infected teeth extracted. Operated by Surgical service. No evidence of ulcer or carcinoma found. A fatty, whitish, enlarged gall-bladder was adherent to duodenum. Palpation of region of appendix gave no evidence of infection. Discharged December 14, 1914, symptomatically well.

H. O. Hospital No. 5695. Male, age 38.

Clinical diagnosis:

1. Pyorrhoea alveolaris.

2. Hypochlorydria with persistent diarrhoea.

No blood, parasites or pus in stool. Mouth was in filthy condition with pyor. rhoea, and several old roots, as noted by Dr. Leonard. Treatment directed to this. Improvement began promptly and persisted.
Mrs. C. C. Hospital No. 5345. Age 23, admitted September 10, 1914.

Clinical diagnosis:

1. Alveolar abscesses.

2. Multiple neuritis.

3. Pelvic cellulitis (old.)

4. Anemia (secondary type.)

Gave history of having had facial neuralgia a year before. Relieved by extraction of upper left molar. Extraction of infected teeth followed by prompt improvement. Notes by Neurological service show impairment of sensibility to touch in both hands and feet. At time of discharge, November 21, 1914, sensation in hands entirely restored and marked improvement in feet.

Mrs. R. R. Hospital No. 4206. Age 49, female.

Clinical diagnosis:

1. Pyorrhoea alveolaris;

2. Sciatic neuritis (bilateral.)

3. Retroversion of uterus.

4. Laceration of cervix.

Radical treatment of pyorrhoea with moderate but complete improvement of neuritis.

J. McG. Hospital No. 4720. Male, age 36. Admitted May 20, 1914; discharged September 10, 1914.

Clinical diagnosis:

1. Chronic tonsillitis and pharyngitis.

2. Alveolar abscesses.

3. Ethmoidal sinusitis.

4. Bronchial asthma.

5. Pulmonary emphysema.

6. Fibrosis, apex left lung.

Extraction of infected teeth by dental service. Streptococcus viridans obtained from apical abscess and autogenous vaccine made. Some general reaction with headache and malaise following administration of vaccine. Marked improvement of all symptoms. 
J. B. Hospital No. 5132. Male, age 43.

Entered with lobar pneumonia with de. layed resolution. Multiple alveolar $a b$ scesses found. While convalescing, areas of mouth sepsis eliminated. Convalescence rapid. Patient left Hospital well.

TABLE IV-SUMMARY OF I, II AND $\| 11$.

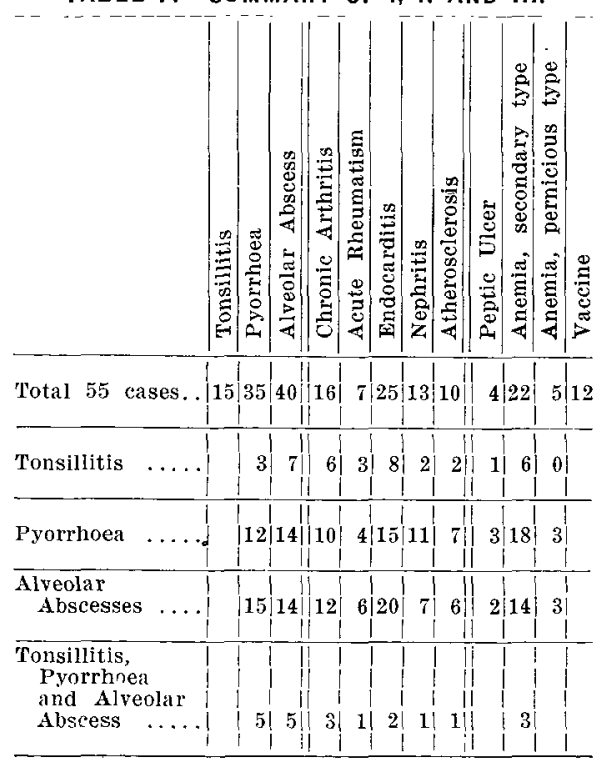

Tables I to IV give the occurrence of

(a). Tonsillitis. Under this head are included all cases with clinical evidences of tonsillar infection, either active or recent, or tonsils with marked scarring and adherence to the pillars of the fauces. In the majority of instances tonsillectomy was requested by the Medical service and carried out by the Nose and Throat service of the Hospital, radical extirpation being done. This was considered an essential step in the management of all cases included in this group, even tho evident dental and peridental infection was found, since the tonsils fail often to give gross evidence of disease, and yet, when adherent and buried, give microscopic and bacteriologic evidence of infection.

Infections in the tonsils and in the teeth are closely related. That tonsillar infection can be, and often is, the source of these dental infections both by continuity of surface and thru the blood stream, seems probable. That the peridental foci may be primary seems also certain. The structural and mechanical peculiarities of each region are such that persistence of infection is invited, physiologic rest is practically impossible, and destruction of infective organisms cannot readily occur. Chronic focal infection is a common result. The needs of the patient demand the elimination of both when diseased, for either may be the source of dissemination, whether primary or secondary.

In the 55 cases included in this study, tonsillitis occurred in 15 , or $27.3 \%$. In 3 it occurred with pyorrhoea, in 7 with alveolar abscess, and in 5 with both.

(b). Pyorrhoea. This includes the cases in which the dental service found clear evidence of pyorrhoeal infection, sometimes limited to one or two teeth, often extensive with large areas of granulation tissue lining the pockets. Of the 55 cases, 35 or $63.6 \%$, showed pyorrhoea, in 12 it occurred alone, in 3 with tonsillitis, in 15 with alveolar abscess, and in 5 with tonsillitis and alveolar abscess.

(c). Alveolar abscesses. This includes 40 of the 55 , or $72.7 \%$ : cases in which Röntgenographic, microscopic, and bacteriologic evidence of abscess were found at the roots of the teeth. In a few instances, the abscess was single: in the majority, multiple: and as high as 6 and 8 abscesses were found by the dental service in the alveolar processes of a single patient.

It is not proposed to discuss here the etiology of alveolar abscess, as that question is foreign to the topic, but a few considerations are of extreme interest and importance to the physician, and 
need mention. Almost without exception the alveolar abscesses are found in devitalized teeth, devitalization having occurred either thru caries with involvement of the pulp canal, or thru artificial devitalization by the dental operator in preparation for crowning or bridge work. The relationship of this devitalization to alveolar abscess appears to be still unsettled. UIrich ${ }^{13}$ believes that the tip of a devitalized tooth becomes a locus minoris resistentiae, and that the infection of the tooth apex is hematogenous in origin.

On the other hand, it seems almost inconceivable that either the death of a tooth pulp thru natural process of caries, or thru artificial destruction with subsequent filling of the pulp chamber, could occur without infection of the tissues about the tooth tip. Examination of radiographs showing the filling material in the pulp chamber of devitalized teeth shows the majority of such fillings to be imperfect, and therefore to invite infection. When to this is added the fact that for the most part dental asepsis has not as yet reached the perfection of the surgical operating rooms, it becomes apparent that the methods used are such as to invite, rather than prevent, infection. These are questions, however, for the dentist, and Dr. Hartzell and his co-workers, with many others in the dental profession are seeking to solve by experimental methods the questions in the etiology of alveolar abscess. It is the existence of such abscesses, the means of their discovery and the systemic disease they cause, that interest the physician.

In reviewing our cases, the first group is that of chronic arthritis of the infective type. Sixteen cases are included.

Concerning chronic arthritis, our knowledge is as yet incomplete. As to the probable points of origin, we most

Ref. 13. H. Id. Ulrich, "Some Medical Aspect; of Certain Mouth Infections." The Dental Review, Dec., 1914. commonly find infections about the teeth, in accessory nasal cavities, the prostate, or the gall-bladder. In the University Hospital the greatest emphasis is placed on the peridental infections. Of the 16 cases, 6 gave evidences of tonsillar infection: 10 had pyorrhoea, 12 had alveolar abscesses with one additional case (No. 5641) showing some absorption of bone about a root tip, 3 had pyorrhoea and abscesses without tonsillar disease, and 3 gave clear evidence of infection of all three regions.

A difficulty in studying results of treatment in this group arises because of the well-known chronicity, the occasional occurrence of long periods of remission, and the relative permanency of periarthritic changes, the integrity of the joint often being more or less permanently impaired, even after the source of infection has been removed. One must guard against hasty conclusions in this field, the only safe criteria of results being the relief from further progress of the lesions with the disappearance of the mild attacks of inflammation. Another source of difficulty is that there may be multiple foci of infection, not all necessarily in one region, and the focus or foci responsible for the disorder may be overlooked, while one or many others have been eliminated.

Among this group of 55 with mouth infection and systemic disease, acute rheumatism occurred 7 times (See Table II), and in only 3 cases was a history or any evidence of tonsillar infection obtainable. Four of the cases had pyorrhoea, and in 6 alveolar abscesses were found. This group is of particular interest because of the generally accepted relationship of tonsillitis to acute rheumatism. The writer is not disposed to be dogmatic here and say that, since evidences of tonsillitis were not found in 4 cases, such tonsillitis did not exist. Experience has shown that it is entirely possible for tonsillar infection to exist deep in the tissues and give only the 
most indefinite symptoms and signs, even with careful study.

Endocarditis occurred in 25, or $45.4 \%$ of the group. (See Tables I, II, and III.) This includes all the cases with evidences of chronic or acute valvular disease. Great significance cannot be attached to this, so far as establishing a relationship to mouth sepsis is concerned, because the persistence of valvular defects makes a permanent record of septic processes which have attacked the endocardium of a given patient up to the time of study. These endocardial involvements occurred in the cases studied under practically all of the groups, they are evidences of a hematogenous infection, and the high percentage of incidence in individuals with mouth infection is very striking, especially when viewed together with experimental evidence to show the affinity of streptococci from dental sources, for cardiovascular tissues. Eight of the cases gave evidence of tonsillar infection. Myocarditis is not included in the tabulation, a clinical diagnosis of this disorder being otten enough made but not often enough proven. In two autopsies, Mrs. L. F., No. 5496 ('Table I) and E. R., No. 3605 (Table II), myocarditis was found. It is to be hoped that accurate polygraphic and electrocardiographic studies will give us more definite information in certain clinical cases thru evidence of altered conduction time when this occurs. In one case, J. B. S., No. 4686 , in which a clinical diagnosis of myocarditis was made, such evidence was found. Pericarditis was found in 3 cases: E. S., No. 4393 , F. I. H., No. 5556, and N. S., No. 4892. (Table II.)

Thirteen instances of nephritis are included, and this occurred usually with other manifestations of disseminated infection. They are of interest, particularly because the experimental work with rabbits has shown distinct tendency for streptococci from dental sources to attack not only the myocardium, endocardium and vascular walls, but the kidney as well. ${ }^{14}$

The group for atherosclerosis is incidental and shows that 10 of the 55 cases, or $18.2 \%$ give clinical evidences of this condition. The number is not large enough to allow statistical analysis, but 4 of these patients were aged 50 years or less.

The work of Rosenow ${ }^{15}$ pointing toward an infection, hematogenous in origin, for gastric ulcer has secured prompt attention. It is wholly in line with established theories as to the causation or probable cause of this as yet obscure disease. Since this cause or probable cause of gastric ulcer has been called to our attention, the number of instances in which septic foci in the mouth are found has increased.

Four of the cases of mouth sepsis had peptic ulcer, and in every instance very prompt subsidence of symptoms followed removal of the dental foci. Careful dieting was not neglected in the care of these patients, but following the apparent cure in the wards, full diet has been recommended, so far without relapse in any of the cases.

Cholecystitis and pyelitis occur frequently in connection with infective processes of the type under discussion, altho seen often enough under conditions where no such focus can be found. No such case should be passed over without attention to the possibility of an infective source in the alveolar processes, tonsils, or elsewhere. One case only, M. O. D. No. 5481, (Table III) with cholecystitis is found here. I have found alveolar abscesses in 3 other cases in as many months.

Ref. 14. Hartzell, "Metastatic Streptococcal Infections Arising from Primary Infections in the Neighborhood of the Human Teeth." Paper prepared for Philadelphia Academy of Stomatology, Nov. 24, 1914.

Ref. 15. Jour. Amer, Med. Ass'n., Vol. LXIII, No. 23, Dec. 5,1914, p. 2027. 
Bissell, ${ }^{16}$ as a result of an extensive experience as $a$ Röntgenologist in the recognition of alveolar and other focal infections suggests the origin of renal colic and renal calculus in a similar manner. He also refers to a large group of cases whose radiographic picture is that of a peribronchial infection somewhat resembling that of tuberculosis, but differing therefrom in certain essential particulars. He says: "We have found dental abscesses in so many of these cases, that I have come to regard them as 'peribronchial streptococcicosis' following Dr. H. L. Ulrich's suggestion as to terminology," and adds "I wish to emphasize the statement that this is an unestablished hypothesis still lacking scientific confirmation."

In our clinic we have now seen a small number of these cases, reacting negatively to tuberculin given subcutaneously, but are not as yet in a position to prove either the bacterial cause for the condition or the relationship to a focal infection elsewhere. These cases are therefore not included in the tabulation.

The anemias of the so-called secondary type are frequently found in connection with infections of all kinds: but it has been only recently that the clinician has learned to reason backward to an infective focus as a probable cause for anemia in obscure cases. The focal infections under consideration are frequently accompanied by a moderate and sometimes severe grade of anemia, altho this anemia is not usually found alone but more commonly accompanies other manifestations of a disseminate 1 infective process. The experience, now frequently repeated, of seeing an anemia lessen or disappear after the elimination of dental and peridental foci of infection leads us now to include such infections among the probable causes.

Of the 55 cases analyzed, 22 or $40 \%$

Ref, 16. The $X$-ray in the Diagnosis of Focal Infections, to be published soon in Journal-Lancet. gave evidences of anemia of the secondary type.

Five cases of anemia of the pernicious type are included. Four (See Table III) are cases in which no other recognizable cause could be found, careful search being made for intestinal parasites and neoplasms, and careful study being made to exclude, if possible, a nephritis sufficient to cause the condition. In one additional case, J. S. No. 5365 (Table II) a nephritis existed, and was looked upon as a probable cause. The results of eradication of dental foci in this group have been disappointing, the improvement occurring being scarcely greater than that so commonly seen in pernicious anemia. The case are included because of the marked oral sepsis present, but the relationship is still obscure.

Isolated instances of ofher conditions are included, such as exophthalmic goitre occurring in a patient with chronic arthritis (Mrs. F. W. No. 3542, Table I): iritis and facial neuralgia, with chronic endocarditis, (Mrs. B. L. No. 4271) : multiple neuritis with old pelvic cellulitis and secondary anemia (Mrs. C. C. No. 5345): sciatic neuritis, bilateral (Mrs. R. R. No. 4206): sinusitis (ethmoidal) (J. McG. No. 4720): lobar pneumonia with delayed resolution (J. B. No. 5132); and hypochlorhydria with persistent diarrhoea (H. O. No. 5694). The inclusion of the above cases may readily be criticized, but in each instance marked oral sepsis was found and the eradication was followed by prompt and striking re. covery. The clinician sees the above types outside of, more frequently than in, the hospital.

It should be noted that in this tabula. tion of 55 cases, as many more cases with mouth infection in our wards have been excluded because of a lack of any definite relationship to the clinical condition. In many of these latter, eradication of foci of infection appeared to allow a prompt increase in resistance with improvement of other apparently unre- 
lated conditions. I have been particularly struck with the marked advantage secured in a number of cases of early, non-ulcerative, so-called "incipient" tuberculosis thru eradication of any existing focal infections, in the mouth or elsewhere. The work of the dental service is now established as a necessity in our Medical Clinic.

In 12 cases of the whole group studied, autogenous vaccines (bacterins) were used, the culture being secured from an abscess or pyorrhoea pocket. All but 3 of these were cases of chronic arthritis. In the majority of instances the injection of the vaccine was followed by an inflammatory reaction about the joints, indicating a specific relationship. No brilliant results, except possibly the cases of A. P. No. 1032 and 2806, and M. B. No. 3732 (both in Table I), have been secured, and the number of cases is too small to use statistically. Vaccines have not been used except in connection with complete eradication of known foci, and it is therefore impossible to claim more than a helpful influence for them. It is hoped to have a much larger series eventually for analysis. In this field, the aid of a competent immunologist must be secured, for many problems of individual resistance and reaction arise, and the dosage must be varie 1 with the character of the case and the nature of the reactions.

The problem of eradication of dental foci of infection differs radically from that presentel in the tonsils. In the ease of the tonsil, the clinical evidences of infection may be difficult to secure. One who has systematically attempted to eradicate focal infections will be often called upon to insist upon the removal of a fairly innocent-looking pair of tonsils even in the face of statements by competent nose and throat surgeons that the tonsils do not appear diseased. We frequently see infection arising from tonsils which are small, buried and adher- ent to the pillars and that show no external sign of inflammation, except possibly a streak of reddening along the pillar. Such tonsils are as frequently the source of systemic dissemination as the frankly and evidently inflamed ones. Where such tonsils exist and where there is no clear evidence of some other focal infection, the need for tonsillectomy rests more upon whether there is evidence of systemic infection from some focus than upon the apparent condition of the tonsil itself. As a result of this attitude, we have been frequently rewarded by having the pathologists, after removal of the tonsils, find definite evidences of infection when clincal evidences of active inflammation were lacking.

At the present time, we have no more definite clinical criteria of infection in the tonsil than I have outlined above. The demonstration of streptococci and other organisms on the surface or in the crypts of tonsils in clinical cases is conclusive only of their existence there. No certain means of securing uncontaminated cultures from the depth of tonsils, clinically, is known to the writer.

The case is very different as concerns the teeth and jaws. Here the dentist can, by proper heat and electricity tests, determine whether teeth are living ones or not: and the Röntgenogram, with proper technic and experience, can give evidences suggestive of infective processes about the teeth or anywhere in the tissues of the jaw. The technic and details of Röntgenographic study are matters for the technician, and a large experience is necessary before a properly qualified opinion can be expressed.

While the ordinary root abscess is easy of recognition, a great deal remains to be learned as to the significance of the minor grades of absorption about the roots. It appears to be true also that in many instances a focus of infection has been absorbed, and resitution of the tissues of the alveolar process has oc- 
curred, leaving a modified Röntgenographic field. The nature and significance of these modifications still remains to be worked out.

It would appear to be a simple matter, once abscesses or infected teeth have been found, to decide what procedure should be adopted: but, on the one hand, the clinician, anxious to eradicate all foci of infection, demands that infected teeth be extracted: the dentist, anxious to retain the best occlusal surfaces and masticating mechanism for the patient, desires to remove only the infected tis. sues and retain as much as possible of the tooth. The application to each individual case should be determined, not by the physician alone, nor by the dentist alone, but by both together giving proper consideration to the needs of the patient, possibility of the dental procedures to eradicate all infection and still retain a masticating surface, and finally, the ability of the individual dental operator involved, so far as securing results in concerned.

Dentists have built up a marvelous mechanical perfection in crown and bridge work, but at the same time have developed conditions inviting infection of the alveolar process. Because so often free from local symptoms and signs, this infection has remained hidden until brought to light by the Röntgenogram. The infection must be eradicated, but so far as possible, our patients must be spared the inconvenience and disability of artificial teeth, and the conservative dentist must learn so far as possible to eradicate the infection and spare the tooth. In this problem the physician has a vital interest.

One additional point needs particular attention by the physician. It is that, if extraction or other operative work is to be employed, care should be exercised not to overdo or to attack too many foci at one time. In this field the infections are usually very chronic, and there is no urgent demand for the immediate eradication of all foci.

Two considerations demand that all foci should not be eradicated at once. The first is that in case vaccines or bacterians are needed, if all foci have been eradicated and attempts at cultivation of bacteria have failed or gone awry, material for culture can no longer be secured.

Secondly, the measures necessary for elimination of the infection frequently stir up and increase the infection at the time and there is considerable danger, particularly in heavily infected individuals, of opening up many channels of infection, of severe local reactions, sometimes with necrosis, and frequently of aggravating a multiple joint infection, or even an endocardial or myocardial involvement. These dangers are real, and we have had several illustrations of the folly of attempting to eradicate multiple foci at one time. Here again it is necessary that the physician and dentist confer and take fully into account such possibilities. 